\title{
Boundedness in a three-dimensional chemotaxis-haptotaxis model
}

\author{
Xinru Cao* \\ Institute for Mathematical Sciences, Renmin University of China, 100872 Beijing, China
}

August 1, 2021

\begin{abstract}
This paper studies the chemotaxis-haptotaxis system

$$
\begin{cases}u_{t}=\Delta u-\chi \nabla \cdot(u \nabla v)-\xi \nabla \cdot(u \nabla w)+\mu u(1-u-w), & (x, t) \in \Omega \times(0, T), \\ v_{t}=\Delta v-v+u, & (x, t) \in \Omega \times(0, T), \\ w_{t}=-v w, & (x, t) \in \Omega \times(0, T)\end{cases}
$$

under Neumann boundary conditions. Here $\Omega \subset \mathbb{R}^{3}$ is a bounded domain with smooth boundary and the parameters $\xi, \chi, \mu>0$. We prove that for nonnegative and suitably smooth initial data $\left(u_{0}, v_{0}, w_{0}\right)$, if $\chi / \mu$ is sufficiently small, $(\star)$ possesses a global classical solution which is bounded in $\Omega \times(0, \infty)$. We underline that the result fully parallels the corresponding parabolic-elliptic-ODE system.
\end{abstract}

\section{Introduction}

The motion of cells moving towards the higher concentration of a chemical signal is called chemotaxis. A classical mathematical model for this type of processes was initiated by Keller and Segel in the 1970's 9], and in a prototypical form this model writes

$$
\begin{cases}u_{t}=\Delta u-\chi \nabla \cdot(u \nabla v), & (x, t) \in \Omega \times(0, T), \\ v_{t}=\Delta v-v+u, & (x, t) \in \Omega \times(0, T) .\end{cases}
$$

Here $u$ is the density of cells, $v$ denotes the concentration of the chemical substance, and $\chi>0$ measures the sensitivity of chemotactic response. In recent 40 years, a large quantity of literature has been devoted to study the global existence as well as singularity formation in either finite or infinite time for this system [10, 17, 21. We also refer to [6, 5, 2] for a broad overview.

Apart from this classical Keller-Segel system, a large number of variants has been proposed to describe taxis phenomena in mathematical biology. Among them, a model for tumor invasion mechanism was introduced by Chaplain and Lolas [3. In this model, tumor cells are assumed to produce a diffusive chemical substance, the so-called matrix-degrading enzyme (MDE), which decays non-diffusive static healthy tissue (ECM). It is observed that both the enzyme and the healthy tissue can attract the cancer cells in the sense that the cancer cells bias their movement along the gradients of the concentrations of both ECM and MDE, where the former of these processes, namely taxis toward a non-diffusible quantity, is usually referred as haptotaxis.

\footnotetext{
*caoxinru@gmail.com, supported by the Fundamental Research Funds for the Central Universities, and the Research Funds of Renmin University of China
} 
Additionally, the cancer cells compete for space with ECM, and at the considered time scales moreover logistic-type cell kinetics need to be taken into account. If futhermore the ability of ECM to spontaneously renew is included, the Chaplain-Lolas model becomes

$$
\begin{cases}u_{t}=\Delta u-\chi \nabla \cdot(u \nabla v)-\xi \nabla \cdot(u \nabla w)+\mu u(1-u-w), & (x, t) \in \Omega \times(0, T), \\ \tau v_{t}=\Delta v-v+u, & (x, t) \in \Omega \times(0, T), \\ w_{t}=-v w+\eta w(1-u-w), & (x, t) \in \Omega \times(0, T),\end{cases}
$$

where $u, v$ and $w$ denote the density of cells, the concentration of MDE and the density of ECM, respectively, where the parameters $\xi, \chi, \mu, \eta$ are positive constants and $\tau \geq 0$, and where $\Omega \subset \mathbb{R}^{N}$, $N \geq 1$, denotes the physical domain under consideration.

Assuming $w \equiv 0,(1.2)$ is reduced to the classical Keller-Segel system with logistic source, which has extensively been studied during the past 20 years. Compared with the pure chemotaxis system mentioned above, one may expect the logistic source and, especially, death terms to enhance the possibility of bounded solutions. In fact, Tello and Winkler [18] proved that if $\tau=0$ and

$$
\mu>\frac{(N-2)_{+}}{N} \chi
$$

then for any regular initial data, the system admits a unique global classical solution which is bounded. In the case $\tau=1$, it is known that bounded solutions exist in lower dimensions $(N=1,2)$ for any $\mu>0$ [8], and that the same result holds for $\mu>\mu_{0}$ with some $\mu_{0}(\chi)>0$ in higher dimensions [20]. More precisely, a careful inspection of the proofs therein shows that in fact large values of the ratio $\frac{\mu}{\chi^{2}}$ are sufficient to exclude blow up in either finite time or infinite time.

Concerning (1.2) with possibly nontrivial $w$, the strong coupling between remodeling and chemotaxis substantially complicates the situation, and accordingly the knowledge on this topic is quite incomplete so far. To the best of our knowledge, global existence of weak solution is obtained in [1] for $N \leq 3$, where (1.2) is included as a subsystem. And global solvability of classical solutions in this full system is known only when $\tau=0$ and $N=2[16]$. Disregarding the chemotaxis effect, the haptotaxis-only version with $\chi=0, \tau=1$ was studied in [12].

In the real situations, the ECM degrades much faster than it renews, thus the remodeling effect can be neglected, that is, we may assume $\eta=0$. Under this hypothesis, the corresponding parabolic-elliptic simplification $\tau=0$ has been studied by Tao and Winkler in [15], where it has been proved that solutions stay bounded under the same condition as in the case $w \equiv 0$, that is, when (1.3) holds. This shows that in this situation the haptotaxis term does not affect the boundedness of solution, and that accordingly the chemotaxis process essentially dominates the whole system. A natural question is whether a similar conclusion holds in the fully parabolic system obtained on letting $\tau=1$. In [13], Tao gives a partially positive answer in this direction by proving that when $N=2$, solutions remain bounded for any $\mu>0$, which thus parallels known results both for $\tau=0$, and also for $\tau=1$ when $w \equiv 0$. As far as we can tell, however, despite a result on global existence established in [14, the question of boundedness of solutions is completely open in higher dimensions. It is the purpose of this work to furthermore establish a corresponding parallel result for the three-dimensional parabolic-parabolic-ODE chemotaxis-haptotaxis model in this direction. 
Accordingly, we deal with the system

$$
\begin{cases}u_{t}=\Delta u-\chi \nabla \cdot(u \nabla v)-\xi \nabla \cdot(u \nabla w)+\mu u(1-u-w), & (x, t) \in \Omega \times(0, T), \\ v_{t}=\Delta v-v+u, & (x, t) \in \Omega \times(0, T), \\ w_{t}=-v w, & (x, t) \in \Omega \times(0, T), \\ \frac{\partial u}{\partial \nu}-\chi u \frac{\partial v}{\partial \nu}-\xi u \frac{\partial w}{\partial \nu}=\frac{\partial v}{\partial \nu}=0, & (x, t) \in \partial \Omega \times(0, T), \\ u(x, 0)=u_{0}(x), \quad v(x, 0)=v_{0}(x), w(x, 0)=w_{0}(x), & x \in \Omega,\end{cases}
$$

where $\Omega \subset \mathbb{R}^{3}$ is bounded with smooth boundary and $\chi, \xi, \mu>0$. We assume that initial data are regular enough and satisfy a standard compatibility condition in the sense that

$$
\left\{\begin{array}{l}
u_{0} \in C^{0}(\bar{\Omega}), \quad v_{0} \in W^{1, \infty}(\Omega), \quad w_{0} \in C^{2, \alpha}(\bar{\Omega})(\alpha \in(0,1)), \\
\frac{\partial w_{0}}{\partial \nu}=0 .
\end{array}\right.
$$

Then our main result says the following.

Theorem 1. There exists $\theta_{0}>0$ such that whenever $\chi>0, \mu>0$ and $\xi>0$ are such that $\frac{\chi}{\mu}<\theta_{0}$, for any initial data $\left(u_{0}, v_{0}, w_{0}\right)$ fulfilling (1.5), there exists a unique classical solution $(u, v, w)$, which is global in time and bounded in $\Omega \times(0, \infty)$.

Remark 1.1. We only carry out the proofs for three-dimensional case in this paper. Actually, we can start the iteration from the higher regularity of $v \in L^{\frac{2 N}{N-2}}(\Omega)$, the same result still holds for $N \leq 15$.

We see that although our hypothesis on the parameters is not as explicit as (1.3) obtained for the parabolic-elliptic counterpart, it still shows that again boundedness of solutions is enforced by a condition merely referring to the interplay between chemotaxis and quadratic degradation in logistic source.

Apart from this, we find it worth mentioning that our approach even shows a new result for the pure fully parabolic chemotaxis system with logistic source in the sense that when $w \equiv 0, N \geq 3$, the system admits a classical bounded solution if $\frac{\mu}{\chi}$ is sufficiently large. Compared with a similar conclusion under the alternative assumption that $\frac{\mu}{\chi^{2}}$ be large [20, our result seems more consistent with (1.3) for the parabolic-elliptic system where the linear ratio $\frac{\mu}{\chi}$ is found to determine the boundedness of solution.

\section{Preliminaries}

We first state a result on local existence of classical solutions. Without essential difficulties, the proof can be derived based on that in [17, Lemma 2.1].

Lemma 2.1. Let $N=3, \chi>0, \xi>0$ and $\mu>0$. For $\left(u_{0}, v_{0}, w_{0}\right)$ satisfying (1.5), there is $T_{\max } \in(0, \infty]$ such that 1.4 admits a unique classical solution

$$
\begin{aligned}
& u \in C^{0}\left(\bar{\Omega} \times\left[0, T_{\max }\right)\right) \cap C^{2,1}\left(\bar{\Omega} \times\left(0, T_{\max }\right)\right), \\
& v \in C^{0}\left(\bar{\Omega} \times\left[0, T_{\max }\right)\right) \cap C^{2,1}\left(\bar{\Omega} \times\left(0, T_{\max }\right)\right) \cap L_{l o c}^{\infty}\left(\left[0, T_{\max }\right) ; W^{1, q}(\Omega)\right)(q>3), \\
& w \in C^{2,1}\left(\bar{\Omega} \times\left[0, T_{\max }\right)\right),
\end{aligned}
$$

such that

$$
u \geq 0, \quad v \geq 0 \quad \text { and } \quad 0<w \leq\left\|w_{0}\right\|_{L^{\infty}(\Omega)} \quad \text { for all } \quad t \in\left[0, T_{\max }\right) .
$$


Moreover, if $T_{\max }<\infty$, then

$$
\limsup _{t \nearrow T_{\max }}\left(\|u(\cdot, t)\|_{L^{\infty}(\Omega)}\right)=\infty .
$$

According to the above existence theory, we know that if we fix any $s_{0} \in\left(0, T_{\max }\right)$, then there exists $M>0$ such that

$$
\left\|u\left(\cdot, s_{0}\right)\right\|_{L^{\infty}(\Omega)}+\left\|v\left(\cdot, s_{0}\right)\right\|_{L^{\infty}(\Omega)}+\left\|w\left(\cdot, s_{0}\right)\right\|_{W^{2, \infty}(\Omega)}<M .
$$

Observing that $w$ can be represented by $v$ and $w\left(x, s_{0}\right)$, we can compute $\Delta w$ in a convenient way. Upon a slight adaptation of [15, Lemma 2.2], we can prove a one-sided pointwise estimate for $\Delta w$ as follows.

Lemma 2.2. Let $\left(u_{0}, v_{0}, w_{0}\right)$ satisfy (1.5), $(u, v, w)$ solve (1.4). For all $s_{0} \geq 0$, we have

$$
\begin{aligned}
\Delta w(x, t) \geq & \Delta w\left(x, s_{0}\right) \cdot e^{-\int_{s_{0}}^{t} v(x, s) d s}-2 e^{-\int_{s_{0}}^{t} v(x, s) d s} \nabla w\left(x, s_{0}\right) \cdot \int_{s_{0}}^{t} \nabla v(x, s) d s \\
& -\frac{1}{e} w\left(x, s_{0}\right)-w\left(x, s_{0}\right) v(x, t) e^{-\int_{s_{0}}^{t} v(x, s) d s}
\end{aligned}
$$

for all $x \in \Omega$ and all $t \in\left(s_{0}, T_{\max }\right)$.

Proof. Representing $w(x, t)$ according to

$$
w=e^{-\int_{s_{0}}^{t} v(x, s) d s} w\left(x, s_{0}\right)
$$

for all $x \in \Omega$ and $t \in\left(s_{0}, T_{\max }\right)$, we directly compute

$$
\begin{aligned}
\Delta w(x, t)= & \Delta w\left(x, s_{0}\right) e^{-\int_{s_{0}}^{t} v(x, s) d s}-2 e^{-\int_{s_{0}}^{t} v(x, s) d s} \nabla w\left(x, s_{0}\right) \cdot \int_{s_{0}}^{t} \nabla v(x, s) d s \\
& +w\left(x, s_{0}\right) e^{-\int_{s_{0}}^{t} v(x, s) d s}\left|\int_{s_{0}}^{t} \nabla v(x, s) d s\right|^{2}-w\left(x, s_{0}\right) e^{-\int_{s_{0}}^{t} v(x, s) d s} \int_{s_{0}}^{t} \Delta v(x, s) d s .
\end{aligned}
$$

Since $z e^{-z} \leq \frac{1}{e}$, by dropping some nonnegative terms, we obtain that

$$
\begin{gathered}
\Delta w(x, t) \geq \Delta w\left(x, s_{0}\right) e^{-\int_{s_{0}}^{t} v(x, s) d s}-2 e^{-\int_{s_{0}}^{t} v(x, s) d s} \nabla w\left(x, s_{0}\right) \cdot \int_{s_{0}}^{t} \nabla v(x, s) d s \\
-w\left(x, s_{0}\right) e^{-\int_{s_{0}}^{t} v(x, s) d s} \int_{s_{0}}^{t}\left(v_{s}(x, s)+v(x, s)-u(x, s)\right) \\
\geq \Delta w\left(x, s_{0}\right) e^{-\int_{s_{0}}^{t} v(x, s) d s}-2 e^{-\int_{s_{0}}^{t} v(x, s) d s} \nabla w\left(x, s_{0}\right) \cdot \int_{s_{0}}^{t} \nabla v(x, s) d s \\
-w\left(x, s_{0}\right) e^{-\int_{s_{0}}^{t} v(x, s) d s}\left(v(x, t)-v\left(x, s_{0}\right)\right)-w\left(x, s_{0}\right) e^{-\int_{s_{0}}^{t} v(x, s) d s} \int_{0}^{t} v(x, s) d s \\
\geq \Delta w\left(x, s_{0}\right) e^{-\int_{s_{0}}^{t} v(x, s) d s}-2 e^{-\int_{s_{0}}^{t} v(x, s) d s} \nabla w\left(x, s_{0}\right) \cdot \int_{0}^{t} \nabla v(x, s) d s \\
-w\left(x, s_{0}\right) v(x, t) e^{-\int_{s_{0}}^{t} v(x, s) d s}-\frac{1}{e} w\left(x, s_{0}\right)
\end{gathered}
$$

for all $t \in\left(s_{0}, T_{\max }\right)$. Thus the proof is complete.

With the aid of Lemma 2.2. we can furthermore prepare a preliminary estimate of an integral related to the haptotactic interaction. This estimate will be used in different ways later on. 
Lemma 2.3. Let $\chi>0, \xi>0$, and assume that (1.5) holds. Then for any $p>1, s_{0} \in\left(0, T_{\max }\right)$, the solution of (1.4) satisfies

$$
(p-1) \xi \int_{\Omega} u^{p-1} \nabla u \cdot \nabla w \leq\left(3 M \xi+\frac{1}{e} M \xi\right) \int_{\Omega} u^{p}+M \xi \int_{\Omega} u^{p} v+2 M(p-1) \xi \int_{\Omega} u^{p-1}|\nabla u|
$$

for all $t \in\left(s_{0}, T_{\max }\right)$.

Proof. Integration by parts and an application of Lemma 2.2 yield that

$$
\begin{aligned}
& (p-1) \xi \int_{\Omega} u^{p-1} \nabla u \cdot \nabla w \\
= & -\frac{p-1}{p} \xi \int_{\Omega} u^{p} \Delta w \\
\leq & -\frac{p-1}{p} \xi \int_{\Omega} u^{p}\left(\Delta w\left(x, s_{0}\right) e^{-\int_{s_{0}}^{t} v(x, s) d s}-2 e^{-\int_{s_{0}}^{t} v(x, s) d s} \nabla w\left(x, s_{0}\right) \cdot \int_{s_{0}}^{t} \nabla v(x, s) d s\right. \\
& \left.\quad-\frac{1}{e} w\left(x, s_{0}\right)-w\left(x, s_{0}\right) v(x, t) e^{-\int_{s_{0}}^{t} v(x, s) d s}\right) \\
\leq & \left(M \xi+\frac{1}{e} M \xi\right) \int_{\Omega} u^{p}+M \xi \int_{\Omega} u^{p} v-2 \frac{p-1}{p} \xi \int_{\Omega} u^{p} \nabla w\left(x, s_{0}\right) \cdot \nabla e^{-\int_{s_{0}}^{t} v(x, s) d s} \\
= & \left(M \xi+\frac{1}{e} M \xi\right) \int_{\Omega} u^{p}+M \xi \int_{\Omega} u^{p} v+2 \frac{p-1}{p} \xi \int_{\Omega} u^{p} \Delta w\left(x, s_{0}\right) e^{-\int_{s_{0}}^{t} v(x, s) d s} \\
& \quad+2 \frac{p-1}{p} \xi \int_{\Omega} \nabla u^{p} \cdot \nabla w\left(x, s_{0}\right) e^{-\int_{s_{0}}^{t} v(x, s) d s} \\
\leq & \left(3 M \xi+\frac{1}{e} M \xi\right) \int_{\Omega} u^{p}+M \xi \int_{\Omega} u^{p} v+2 M(p-1) \xi \int_{\Omega} u^{p-1}|\nabla u|
\end{aligned}
$$

for all $t \in\left(s_{0}, T_{\max }\right)$, where in accordance with (2.3), $M$ is an upper bound for $\left\|w\left(\cdot, s_{0}\right)\right\|_{W^{2, \infty}(\Omega)}$.

Lemma 2.4. Let $\chi>0, \xi>0$ and $\mu>0$, and assume (1.5). Then there exists $C(\mu,|\Omega|)>0$ such that

$$
\int_{\Omega} u(\cdot, t)<C, \quad \int_{\Omega} v(\cdot, t)<C
$$

for all $t \in\left(0, T_{\max }\right)$.

Proof. The first inequality in (1.4) can be proved by simply integrating the first equation in (1.4) on $\Omega$ and using that $\left(\int_{\Omega} u\right)^{2} \leq|\Omega|\left(\int_{\Omega} u^{2}\right)$ due to the Cauchy-Schwarz inequality. The estimate of $\int_{\Omega} v$ can be obtained in a similar way and with the aid of the first inequality.

As an essential ingredient of the proof of our main result, we will use a Maximal Sobolev regularity property associated with the second equation in (1.4). The following lemma is not the original version of a corresponding statement in [4, Theorem 3.1], but by means of a simple transformation it is adapted to the current situation by including an exponential weight function.

Lemma 2.5. Let $r \in(1, \infty), T \in(0, \infty)$. Consider the following evolution equation

$$
\begin{cases}v_{t}=\Delta v-v+f, & (x, t) \in \Omega \times(0, T), \\ \frac{\partial v}{\partial \nu}=0, & (x, t) \in \partial \Omega \times(0, T), \\ v(x, 0)=v_{0}(x), & x \in \Omega .\end{cases}
$$

For each $v_{0} \in W^{2, r}(\Omega)$ such that $\frac{\partial v_{0}}{\partial \nu}=0$ on $\partial \Omega$ and any $f \in L^{r}\left((0, T) ; L^{r}(\Omega)\right)$, there exists a unique solution

$$
v \in W^{1, r}\left((0, T) ; L^{r}(\Omega)\right) \cap L^{r}\left((0, T) ; W^{2, r}(\Omega)\right),
$$


and there exists $C_{r}>0$ such that

$$
\int_{0}^{T} \int_{\Omega} e^{\frac{1}{2} r s}|\Delta v(x, s)|^{r} d x d s \leq C_{r} \int_{0}^{T} \int_{\Omega} e^{\frac{1}{2} r s}|f(x, s)|^{r} d x d s+C_{r}\left\|v_{0}\right\|_{L^{r}(\Omega)}^{r}+C_{r}\left\|\Delta v_{0}\right\|_{L^{r}(\Omega)}^{r} .
$$

Moreover, if for some $s_{0} \in(0, T), v\left(\cdot, s_{0}\right)$ satisfies $v\left(\cdot, s_{0}\right) \in W^{2, r}(\Omega)$ with $\frac{\partial v}{\partial \nu}(\cdot, s)=0$ on $\partial \Omega$, then with the same constant $C>0$ as above we have

$\int_{s_{0}}^{T} \int_{\Omega} e^{\frac{1}{2} r s}|\Delta v(x, s)|^{r} d x d s \leq C_{r} \int_{s_{0}}^{T} \int_{\Omega} e^{\frac{1}{2} r s}|f(x, s)|^{r} d x d s+C_{r}\left\|v\left(\cdot, s_{0}\right)\right\|_{L^{r}(\Omega)}^{r}+C_{r}\left\|\Delta v\left(\cdot, s_{0}\right)\right\|_{L^{r}(\Omega)}^{r}(2.1$

Proof. Letting $w(x, s)=e^{\frac{1}{2} s} v(x, s)-\chi(s) v_{0}$, where $\chi(s)$ is a cut-off function such that $\chi \in C_{0}^{\infty}([0,1))$, and $\chi(0)=1$, we see that $w$ solves

$$
\left\{\begin{array}{lc}
w_{s}(x, s)=\left(\Delta-\frac{1}{2}\right) w(x, s)+e^{\frac{1}{2} s} f(x, s)+\chi(s) \Delta v_{0}-\frac{1}{2} \chi(s) v_{0}-\chi^{\prime}(s) v_{0}, & (x, s) \in \Omega \times(0, T), \\
\frac{\partial w}{\partial \nu}=0, & (x, s) \in \partial \Omega \times[0, T), \\
w(x, 0)=0, & x \in \Omega .
\end{array}\right.
$$

Let $A$ be $\Delta-\frac{1}{2}$ associated with Neumann boundary condition, we know that $A$ is a generator of an analytic semigroup of negative exponential type. An application of standard results on maximal sobolev regularity provides $c_{r}>0$ such that

$$
\begin{aligned}
& \int_{0}^{T}\|w(\cdot, s)\|_{L^{r}}^{r} d s+\int_{0}^{T}\left\|w_{s}(\cdot, t)\right\|_{L^{r}}^{r} d s+\int_{0}^{T}\|A w(\cdot, s)\|_{L^{r}(\Omega)}^{r} d s \\
& \leq c_{r} \int_{0}^{T}\left\|e^{\frac{1}{2} t} f(\cdot, t)\right\|_{L^{r}}^{r}+\left\|\chi(s) \Delta v_{0}-\frac{1}{2} \chi(s) v_{0}-\chi^{\prime}(s) v_{0}\right\|_{L^{r}}^{r} d s \\
& \leq c_{r} \int_{0}^{T}\left\|e^{\frac{1}{2} t} f(\cdot, t)\right\|_{L^{r}}^{r}+c_{r} c_{1}\left\|v_{0}\right\|_{W^{2, r}(\Omega)},
\end{aligned}
$$

where $c_{1}:=2+\sup _{s \in[0,1]} \chi^{\prime}(s)$. The triangle inequality with (2.11) implies that

$$
\begin{aligned}
\int_{0}^{T}\|\Delta w(\cdot, s)\|_{L^{r}}^{r} d s & \leq \int_{0}^{T}\left\|\left(\Delta-\frac{1}{2}\right) w(\cdot, t)\right\|_{L^{r}}^{r} d s+\int_{0}^{T} \frac{1}{2}\|w(\cdot, t)\|_{L^{r}}^{r} d s \\
& \leq c_{r} \int_{0}^{T}\left\|e^{\frac{1}{2} t} f(\cdot, s)\right\|_{L^{r}}^{r} d s+c_{1} c_{r}\left\|v_{0}\right\|_{W^{2, r},}^{r},
\end{aligned}
$$

which leads to (2.9) upon direct computation and letting $C_{r}:=\max \left\{c_{r}, c_{1} c_{r}\right\}$. Thereafter, (2.10) directly follows upon replacing $v(x, t)$ by $v\left(x, t+s_{0}\right)$, where the constant might be different from the aforementioned one.

\section{Boundedness}

In this section, we derive the claimed boundedness result via combining the above result on maximum Sobolev regularity with a Moser-type iteration. The former ingredient is first used to estimate $u$ in some appropriate Lebesgue space, from which a certain suitable estimate of $\nabla v$ will follow. This approach will be carried out twice to ensure that $\nabla v$ is bounded in $L^{\infty}(\Omega)$. Thereupon we can establish a series of inequalities based on which a Moser iteration is performed to finally achieve boundedness of $u$ in $L^{\infty}(\Omega)$.

Lemma 3.1. Let $\chi>0, \xi>0, \mu>0, \theta=\frac{\chi}{\mu}$ and let $p_{1} \in\left(\frac{3}{2}, 2\right), p_{2} \in(3, \infty)$. Then there exists $\theta_{0}>0$ such that whenever $\theta<\theta_{0}$, for any $\left(u_{0}, v_{0}, w_{0}\right)$ fulfilling (1.5) and some $s_{0} \in\left(0, T_{\max }\right)$, we can find $C>0$ such that

$$
\int_{\Omega} u^{p_{2}}(\cdot, t) \leq C \quad \text { for all } t \in\left(s_{0}, T_{\max }\right)
$$


We are going to prove Lemma 3.1 by several steps. Let us first provide an important ingredient for the estimate of $\|u(\cdot, t)\|_{L^{p}(\Omega)}$. The next Lemma offers a general iteration step from $v$ to $u$. We will use it to first estimate $\|u(\cdot, t)\|_{L^{p_{1}}(\Omega)}$ with $p_{1} \in\left(\frac{3}{2}, 2\right)$, and then $\|u(\cdot, t)\|_{L^{p_{2}(\Omega)}}$ with $p_{2} \in(3, \infty)$. These results are under different conditions on $\frac{\chi}{\mu}$. For convenience, we assume that both of the conditions hold in Lemma 3.1 such that Lemma 3.2 is applicable for both $p_{1}$ and $p_{2}$.

Lemma 3.2. Let $\chi>0, \xi>0, \mu>0, p>1$. Then there exist constants $\theta_{p}>0$ and $C>0$ such that if $\theta=\frac{\chi}{\mu}<\theta_{p}$ and

$$
\int_{\Omega} v^{p+1}(\cdot, t) \leq C \quad \text { for all } t \in\left(s_{0}, T_{\max }\right)
$$

Then we have

$$
\int_{\Omega} u^{p}(\cdot, t) \leq C \quad \text { for all } t \in\left(s_{0}, T_{\max }\right) .
$$

Proof. First we see that for any $a, b>0$, Young's inequality provides $k_{p}>0$ such that

$$
a b \leq \frac{1}{8} a^{\frac{p+1}{p}}+k_{p} b^{p+1} .
$$

Let $C_{r+1}$ denote the constant from Lemma2.5 for $r \in(1, \infty)$. Now we can find $\theta_{p}>0$ small enough such that

$$
C_{p+1} k_{p} \theta^{p+1} \leq \frac{1}{2} \text { for all } \theta<\theta_{p}
$$

Testing the first equation in (1.4) with $u^{p-1}(p>1)$ and integrating by part imply

$$
\begin{aligned}
& \frac{1}{p} \frac{d}{d t} \int_{\Omega} u^{p}+(p-1) \int_{\Omega} u^{p-2}|\nabla u|^{2} \\
= & (p-1) \chi \int_{\Omega} u^{p-1} \nabla u \cdot \nabla v+(p-1) \xi \int_{\Omega} u^{p-1} \nabla u \cdot \nabla w \\
& \quad+\mu \int_{\Omega} u^{p}-\mu \int_{\Omega} u^{p+1}-\mu \int_{\Omega} u^{p} w \\
\leq & \frac{p-1}{p} \chi \int_{\Omega} \nabla u^{p} \cdot \nabla v+(p-1) \xi \int_{\Omega} u^{p-1} \nabla u \cdot \nabla w+\mu \int_{\Omega} u^{p}-\mu \int_{\Omega} u^{p+1} \\
\leq & -\frac{p-1}{p} \chi \int_{\Omega} u^{p} \Delta v+(p-1) \xi \int_{\Omega} u^{p-1} \nabla u \cdot \nabla w+\mu \int_{\Omega} u^{p}-\mu \int_{\Omega} u^{p+1}
\end{aligned}
$$

for all $t \in\left(s_{0}, T_{\max }\right)$. We see that (2.6) and (3.17) entail the existence of $c_{3}>0$ satisfying

$$
\begin{aligned}
& (p-1) \xi \int_{\Omega} u^{p-1} \nabla u \cdot \nabla w \\
\leq & c_{3} \xi \int_{\Omega} u^{p}+c_{3} \xi \int_{\Omega} u^{p} v+c_{3} p \xi \int_{\Omega} u^{p-1}|\nabla u| \\
\leq & c_{3} \xi \int_{\Omega} u^{p}+\frac{\mu}{8} \int_{\Omega} u^{p+1}+k_{p} c_{3}^{p+1} \mu^{-p} \xi^{p+1} \int_{\Omega} v^{p+1}+\frac{p-1}{2} \int_{\Omega} u^{p-2}|\nabla u|^{2}+\frac{c_{3}^{2} \xi^{2} p^{2}}{2(p-1)} \int_{\Omega} u^{p} \\
\leq & \left(c_{3} \xi+\frac{c_{3}^{2} \xi^{2} p^{2}}{2(p-1)}\right) \int_{\Omega} u^{p}+\frac{\mu}{8} \int_{\Omega} u^{p+1}+\frac{p-1}{2} \int_{\Omega} u^{p-2}|\nabla u|^{2}+k_{p} \mu^{-p} \xi^{p+1}\|v(\cdot, t)\|_{L^{p+1}(\Omega)}^{p+1}
\end{aligned}
$$

for all $t \in\left(s_{0}, T_{\max }\right)$. From (3.4), we estimate for all $t \in\left(s_{0}, T_{\max }\right)$,

$$
-\frac{p-1}{p} \chi \int_{\Omega} u^{p} \Delta v \leq \chi \int_{\Omega} u^{p}|\Delta v| \leq \frac{\mu}{8} \int_{\Omega} u^{p+1}+k_{p} \chi^{p+1} \mu^{-p} \int_{\Omega}|\Delta v|^{p+1} .
$$


Inserting (3.7) and (3.8) into (3.6) and some rearrangement yield

$$
\begin{aligned}
& \frac{1}{p} \frac{d}{d t} \int_{\Omega} u^{p}+\frac{p-1}{2} \int_{\Omega} u^{p-2}|\nabla u|^{2} \\
\leq & -\frac{3}{4} \mu \int_{\Omega} u^{p+1}+\left(c_{3} \xi+\frac{c_{3}^{2} \xi^{2} p^{2}}{2(p-1)}+\mu\right) \int_{\Omega} u^{p}+k_{p} \mu^{-p} \chi^{p+1} \int_{\Omega}|\Delta v|^{p+1}+k_{p} \mu^{-p} \xi^{p+1}\|v(\cdot, t)\|_{L^{p+1}(\Omega)}^{p+1} \\
= & -\frac{p+1}{2 p} \int_{\Omega} u^{p}+\left(\frac{p+1}{2 p}+c_{3} \xi+\frac{c_{3}^{2} \xi^{2} p^{2}}{2(p-1)}+\mu\right) \int_{\Omega} u^{p}-\frac{3}{4} \mu \int_{\Omega} u^{p+1} \\
& +k_{p} \chi^{p+1} \mu^{-p} \int_{\Omega}|\Delta v|^{p+1}+k_{p} \mu^{-p} \xi^{p+1}\|v(\cdot, t)\|_{L^{p+1}(\Omega)}^{p+1}
\end{aligned}
$$

for all $t \in\left(s_{0}, T_{\max }\right)$. We again apply Young's inequality to obtain that

$$
\left(\frac{p+1}{2 p}+c_{3} \xi+\frac{c_{3}^{2} \xi^{2} p^{2}}{2(p-1)}+\mu\right) \int_{\Omega} u^{p} \leq \frac{\mu}{4} \int_{\Omega} u^{p+1}+c_{4}(\mu, \xi, p),
$$

where $c_{5}>0$ depends on $\mu, \xi, p$. Upon (3.9) and (3.10), we infer that

$$
\begin{array}{r}
\frac{d}{d t} \int_{\Omega} u^{p} \leq-\left(\frac{p+1}{2}\right) \int_{\Omega} u^{p}-\frac{\mu}{2} p \int_{\Omega} u^{p+1}+p k_{p} \chi^{p+1} \mu^{-p} \int_{\Omega}|\Delta v|^{p+1} \\
+c_{4}(\mu, \xi, p) p+p k_{p} \mu^{-p} \xi^{p+1}\|v(\cdot, t)\|_{L^{p+1}(\Omega)}^{p+1}
\end{array}
$$

for all $t \in\left(s_{0}, T_{\max }\right)$. Applying Gronwall inequality in different form to the above inequality shows that

$$
\begin{aligned}
\int_{\Omega} u^{p}(\cdot, t) \leq & e^{-\left(\frac{p+1}{2}\right)\left(t-s_{0}\right)} \int_{\Omega} u^{p}\left(\cdot, s_{0}\right)-\frac{\mu}{2} p \int_{s_{0}}^{t} e^{-\left(\frac{p+1}{2}\right)(t-s)} \int_{\Omega} u^{p+1}(\cdot, s) d s \\
& +k p \chi^{p+1} \mu^{-p} \int_{s_{0}}^{t} e^{-\left(\frac{p+1}{2}\right)(t-s)} \int_{\Omega}|\Delta v(\cdot, s)|^{p+1} d s+\int_{s_{0}}^{t} e^{-\left(\frac{p+1}{2}\right)(t-s)}\left(c_{4}(\mu, \xi, p)\right. \\
& \left.+k_{p} \mu^{-p} \xi^{p+1}\|v(\cdot, t)\|_{L^{p+1}(\Omega)}^{p+1}\right) d s \\
= & e^{-\left(\frac{p+1}{2}\right)\left(t-s_{0}\right)} \int_{\Omega} u^{p}\left(\cdot, s_{0}\right)-\frac{\mu}{2} p e^{-\left(\frac{p+1}{2}\right) t} \int_{s_{0}}^{t} \int_{\Omega} e^{\left(\frac{p+1}{2}\right) s} u^{p+1}(\cdot, s) \\
& +p k_{p} \chi^{p+1} \mu^{-p} e^{-\left(\frac{p+1}{2}\right) t} \int_{s_{0}}^{t} \int_{\Omega} e^{\left(\frac{p+1}{2}\right) s}|\Delta v(\cdot, s)|^{p+1} \\
& +\frac{p}{p+1}\left(c_{4}(\mu, \xi, p)+k_{p} \mu^{-p} \xi^{p+1}\|v(\cdot, t)\|_{L^{p+1}(\Omega)}^{p+1}\right)
\end{aligned}
$$

for all $t \in\left(s_{0}, T_{\max }\right)$. In order to estimate the third term therein, let us note that an application of Lemma 2.5 results in

$$
\begin{aligned}
& p k_{p} \chi^{p+1} \mu^{-p} e^{-\left(\frac{p+1}{2}\right) t} \int_{s_{0}}^{t} \int_{\Omega} e^{\left(\frac{p+1}{2}\right) s}|\Delta v|^{p+1} \\
\leq & p k_{p} C_{p+1} \chi^{p+1} \mu^{-p} e^{-\left(\frac{p+1}{2}\right) t} \int_{s_{0}}^{t} \int_{\Omega} e^{\left(\frac{p+1}{2}\right) s} u^{p+1}+C_{p+1} p k_{p} \chi^{p+1} \mu^{-p} e^{-\left(\frac{p+1}{2}\right) t}\left\|v\left(\cdot, s_{0}\right)\right\|_{W^{2, p+1}(\Omega)}^{p+1} \\
= & p k_{p} C_{p+1} \chi^{p+1} \mu^{-p} e^{-\left(\frac{p+1}{2}\right) t} \int_{s_{0}}^{t} \int_{\Omega} e^{\left(\frac{p+1}{2}\right) s} u^{p+1}+C_{p+1} p k_{p} \chi^{p+1} \mu^{-p} e^{-\left(\frac{p+1}{2}\right) t} M^{p+1}
\end{aligned}
$$

for all $t \in\left(s_{0}, T_{\max }\right)$ and $M$ as in (2.3). Since $\theta=\frac{\chi}{\mu}$, combining (3.11]3.12), we finally arrive at

$$
\begin{gathered}
\int_{\Omega} u^{p}(\cdot, t) \leq e^{-\left(\frac{p+1}{2}\right)\left(t-s_{0}\right)} \int_{\Omega} u^{p}\left(\cdot, s_{0}\right)-\mu p\left(\frac{1}{2}-k_{p} C_{p+1} \theta^{p+1}\right) e^{-\left(\frac{p+1}{2}\right) t} \int_{s_{0}}^{t} \int_{\Omega} e^{\left(\frac{p+1}{2}\right) s} u^{p+1} \\
+C_{p+1} p k_{p} \chi^{p+1} \mu^{-p} e^{-\left(\frac{p+1}{2}\right) t} M^{p+1}
\end{gathered}
$$




$$
+\frac{p}{p+1}\left(c_{4}(\mu, \xi, p)+k_{p} \mu^{-p} \xi^{p+1}\|v(\cdot, t)\|_{L^{p+1}(\Omega)}^{p+1}\right)
$$

for all $t \in\left(s_{0}, T_{\max }\right)$. We see from (3.2) and the condition on $\theta_{p}$ in (3.5) that

$$
\int_{\Omega} u^{p}(\cdot, t) \leq C\left(\mu, \chi, \xi, p,\left\|v\left(\cdot, s_{0}\right)\right\|_{W^{2, p+1}(\Omega)}\right)
$$

for all $t \in\left(s_{0}, T_{\max }\right)$ upon obvious choice of $C>0$. Thus the assertion is derived.

Now we can apply Lemma 3.2 to improve the regularity of $u$ from $L^{1}(\Omega)$ to $L^{p_{1}}(\Omega)$.

Lemma 3.3. Let $p_{1} \in\left(\frac{3}{2}, 2\right)$ and $\theta<\theta_{p_{1}}$, there is a constant $C>0$ such that

$$
\|u(\cdot, t)\|_{L^{p_{1}}(\Omega)} \leq C \quad \text { for all } t \in\left(s_{0}, T_{\max }\right) .
$$

Proof. First we use the representation formula for $v$,

$$
v(\cdot, t)=e^{t \Delta} v_{0}+\int_{0}^{t} e^{(t-s)(\Delta-1)} u(\cdot, s) d s
$$

for all $t \in\left(0, T_{\max }\right)$. Let $p_{1} \in\left(\frac{3}{2}, 2\right)$, the $L^{p}-L^{q}$ estimates for the Neumann heat semigroup 19, Lemma1.3] and (2.7) allow us to pick constants $c_{1}>0$ and $c_{2}>0$ such that

$$
\begin{aligned}
\|v(\cdot, t)\|_{L^{p_{1}+1}(\Omega)} & \leq\left\|e^{t(\Delta-1)} v_{0}\right\|_{L^{p_{1}+1}(\Omega)}+\int_{0}^{t}\left\|e^{(t-s)(\Delta-1)} u(\cdot, s)\right\|_{L^{p_{1}+1}(\Omega)} d s \\
& \leq e^{-t}\left\|v_{0}\right\|_{L^{p_{1}+1}(\Omega)}+c_{1} \int_{0}^{t}\left(1+(t-s)^{-\frac{3}{2}\left(1-\frac{1}{p_{1}+1}\right)}\right) e^{-(t-s)}\|u(\cdot, s)\|_{L^{1}(\Omega)} d s \\
& \leq c_{2}
\end{aligned}
$$

for all $t \in\left(0, T_{\max }\right)$. An application of Lemma 3.2 and (3.17) lead to (3.15).

With the higher regularity for $v$ obtained in the above lemma, a similar reasoning for Lemma 3.2 will provide us higher regularity for $u$. Now we are ready to prove Lemma 3.1

Proof of Lemma 3.1. Let $p_{1}$ be choosen as in Lemma 3.2 and $p_{2} \in(3, \infty)$, define $\theta_{0}=\min \left\{\theta_{p_{1}}, \theta_{p_{2}}\right\}$. According to Lemma 3.2 and standard estimates for Neumann heat semigroup generated by $\Delta$, we see that

$$
\begin{aligned}
\|v(\cdot, t)\|_{L^{\infty}(\Omega)} & \leq\left\|e^{t(\Delta-1)} v\left(\cdot, s_{0}\right)\right\|_{L^{\infty}(\Omega)}+\int_{0}^{t}\left\|e^{(t-s)(\Delta-1)} u(\cdot, s)\right\|_{L^{\infty}(\Omega)} d s \\
& \leq e^{-t}\left\|v\left(\cdot, s_{0}\right)\right\|_{L^{\infty}(\Omega)}+c_{1} \int_{s_{0}}^{t}\left(1+(t-s)^{-\frac{3}{2 p_{1}}}\right) e^{-(t-s)}\|u(\cdot, s)\|_{L^{p_{1}}(\Omega)} d s
\end{aligned}
$$

for all $t \in\left(s_{0}, T_{\max }\right)$ with some $c_{1}>0$. Lemma 3.3 guarantees the boundedness of the above estimate. Also Hölder's inequality yields that

$$
\|v(\cdot, t)\|_{L^{p_{2}+1}(\Omega)}<c_{1}, \quad t \in\left(s_{0}, T_{\max }\right) .
$$

Due to the definition of $\theta_{0}$ and (3.19), now we can apply Lemma 3.2 to find the existence of a constant $C\left(\mu, \chi, \xi, p_{2},\left\|v\left(\cdot, s_{0}\right)\right\|_{W^{2, p_{2}+1}(\Omega)}\right)>0$ such that

$$
\int_{\Omega} u^{p_{2}}(\cdot, t) \leq C\left(\mu, \chi, \xi, p_{2},\left\|v\left(\cdot, s_{0}\right)\right\|_{W^{2, p_{2}+1}(\Omega)}\right)
$$

for all $t \in\left(s_{0}, T_{\max }\right)$. Thus (3.3) is obtained. 
An immediate consequence of Lemma 3.1 is that $\nabla v$ is bounded with respect to the norm in $L^{\infty}(\Omega)$.

Lemma 3.4. Under the assumptions of Lemma 3.2, for some $s_{0} \in\left(0, T_{\max }\right)$, there is $C>0$ such that

$$
\|\nabla v(\cdot, t)\|_{L^{\infty}(\Omega)}<C \quad \text { for all } t \in\left(s_{0}, T_{\max }\right) .
$$

Proof. We note that the standard estimate for Neumann semigroup provides $c_{1}>0$ such that

$$
\begin{aligned}
\|\nabla v(\cdot, t)\|_{L^{\infty}(\Omega)} & \leq\left\|\nabla e^{t(\Delta-1)} v\left(\cdot, s_{0}\right)\right\|_{L^{\infty}(\Omega)}+\int_{s_{0}}^{t}\left\|\nabla e^{(t-s)(\Delta-1)} u(\cdot, s)\right\|_{L^{\infty}(\Omega)} \\
& \leq e^{-t}\left\|\nabla v\left(\cdot, s_{0}\right)\right\|_{L^{\infty}(\Omega)}+\int_{s_{0}}^{t} c_{1}\left(1+(t-s)^{-\frac{1}{2}-\frac{3}{2 p_{2}}}\right) e^{-(t-s)}\|u(\cdot, s)\|_{L^{p_{2}(\Omega)}} d s
\end{aligned}
$$

for all $t \in\left(0, T_{\max }\right)$. In light of Lemma 3.1 we see the conclusion is established.

Now we are in a position to prove boundednes of $u$. Since the estimate of $\nabla w$ is nonlocal in time, the Moser iteration procedure is still necessary. Upon which, we can finally show that $u$ is bounded.

Lemma 3.5. Under the assumptions of Lemma 3.2, for some $s_{0} \in\left(0, T_{\max }\right)$, there is $C>0$ such that

$$
\|u(\cdot, t)\|_{L^{\infty}(\Omega)} \leq C \quad \text { for all } t \in\left(s_{0}, T_{\max }\right)
$$

Proof. We first see Lemma 3.3 and Lemma 3.4 imply the existence of $c_{1}>0$ such that

$$
\|v(\cdot, t)\|_{L^{\infty}(\Omega)}+\|\nabla v(\cdot, t)\|_{L^{\infty}(\Omega)}<c_{1}
$$

for all $t \in\left(s_{0}, T_{\max }\right)$. Testing the first equation in (1.4) with $u^{p-1}(p>1)$, using (2.6), (3.20), (3.18) and Young's inequality, we can find constants $c_{2}, c_{3}>0$ such that

$$
\begin{aligned}
& \frac{1}{p} \frac{d}{d t} \int_{\Omega} u^{p}+(p-1) \int_{\Omega} u^{p-2}|\nabla u|^{2} \\
= & (p-1) \chi \int_{\Omega} u^{p-1} \nabla u \cdot \nabla v+(p-1) \xi \int_{\Omega} u^{p-1} \nabla u \cdot \nabla w+\mu \int_{\Omega} u^{p}-\mu \int_{\Omega} u^{p+1}-\mu \int_{\Omega} u^{p} w \\
\leq & (p-1) \chi \int_{\Omega} u^{p-1} \nabla u \cdot \nabla v+c_{2} \xi \int_{\Omega} u^{p}+c_{2} \xi \int_{\Omega} u^{p} v+c_{2} \xi \int_{\Omega} u^{p-1}|\nabla u|+\mu \int_{\Omega} u^{p} \\
\leq & \frac{p-1}{4} \int_{\Omega} u^{p-2}|\nabla u|^{2}+(p-1) \chi^{2} \int_{\Omega} u^{p}|\nabla v|^{2}+c_{2} \xi \int_{\Omega} u^{p}+c_{1} c_{2} \xi \int_{\Omega} u^{p} \\
& +\frac{p-1}{4} \int_{\Omega} u^{p-2}|\nabla u|^{2}+c_{2}^{2} \xi^{2} p \int_{\Omega} u^{p}+\mu \int_{\Omega} u^{p} \\
\leq & \frac{p-1}{2} \int_{\Omega} u^{p-2}|\nabla u|^{2}+c_{3} p \int_{\Omega} u^{p}
\end{aligned}
$$

for all $t \in\left(s_{0}, T_{\max }\right)$. An obvious rearrangement implies

$$
\frac{d}{d t} \int_{\Omega} u^{p}+c_{4} \int_{\Omega}\left|\nabla u^{\frac{p}{2}}\right|^{2} \leq c_{5} p^{2} \int_{\Omega} u^{p}
$$

for all $t \in\left(s_{0}, T_{\max }\right)$ where $c_{4}, c_{5}>0$ are independent of $p$. Next, we use (3.23) to perform the classical Moser iteration procedure [1] to obtain the boundedness of $u$.

Let $p_{k}=2^{k}, k \in \mathbb{N}$ and $M_{k}:=\sup _{t \in\left(s_{0}, T_{\max }\right)} \int_{\Omega} u^{p_{k}}(\cdot, t)$. Since $p_{k} \geq 1$, it is easy to find $c_{6}>0$ such that

$$
\frac{d}{d t} \int_{\Omega} u^{p_{k}}+\int_{\Omega} u^{p_{k}}+c_{4} \int_{\Omega}\left|\nabla u^{\frac{p_{k}}{2}}\right|^{2} \leq c_{5} p_{k}^{2} \int_{\Omega} u^{p_{k}}+\int_{\Omega} u^{p_{k}} \leq c_{6} p_{k}^{2} \int_{\Omega} u^{p_{k}}
$$


for all $t \in\left(s_{0}, T_{\max }\right)$. By means of the Gargliardo-Nirenberg inequality, we get that

$$
\int_{\Omega} u^{p_{k}}=\left\|u^{\frac{p_{k}}{2}}\right\|_{L^{2}(\Omega)}^{2} \leq c_{7}\left\|\nabla u^{\frac{p_{k}}{2}}\right\|_{L^{2}(\Omega)}^{2 a}\left\|u^{\frac{p_{k}}{2}}\right\|_{L^{1}(\Omega)}^{2(1-a)}+c_{7}\left\|u^{\frac{p_{k}}{2}}\right\|_{L^{1}(\Omega)}^{2}
$$

with $a=\frac{\frac{N}{2}}{1+\frac{N}{2}} \in(0,1)$ and some $c_{7}>0$ independent of $k$. Young's inequality and the definition of $p_{k}$ ensure that there are $c_{8}>0$ and $b>0$ satisfying

$$
\begin{aligned}
c_{6} p_{k}^{2} \int_{\Omega} u^{p_{k}} & \leq c_{4} \int_{\Omega}\left|\nabla u^{\frac{p_{k}}{2}}\right|^{2}+c_{8}\left(p_{k}^{2}\right)^{\frac{1}{1-a}}\left(\int_{\Omega} u^{p_{k-1}}\right)^{2}+c_{6} c_{7} p_{k}^{2}\left(\int_{\Omega} u^{p_{k-1}}\right)^{2} \\
& \leq c_{4} \int_{\Omega}\left|\nabla u^{\frac{p_{k}}{2}}\right|^{2}+b^{k} M_{k-1}^{2} .
\end{aligned}
$$

Combining 3.24 3.25 find that

$$
\frac{d}{d t} \int_{\Omega} u^{p_{k}}+\int_{\Omega} u^{p_{k}} \leq b^{k} M_{k-1}^{2}
$$

for all $t \in\left(s_{0}, T_{\max }\right)$. The comparison theorem for the above ODE yields

$$
M_{k} \leq \max \left\{b^{k} M_{k-1}^{2}, \int_{\Omega} u^{p_{k}}\left(\cdot, s_{0}\right)\right\} .
$$

If $b^{k} M_{k-1}^{2}<\int_{\Omega} u^{p_{k}}\left(\cdot, s_{0}\right)$ is valid for infinite $k$, 3.5 is already derived. Otherwise, a direct induction entails

$$
M_{k} \leq b^{k} M_{k-1}^{2} \leq b^{\sum_{j=1}^{j=k-1} 2^{j}(k-j)} M_{0}^{2 k} .
$$

Taking $2^{k}$-th root on both sides leads to the assertion.

Now we are ready to prove Theorem 1 .

Proof of Theorem 1. First we see that the boundedness of $u$ and $v$ follow from Lemma 3.5. Lemma 3.3 and (2.3). Thereupon the assertion of Theorem 1 is immediately obtained from Lemma 2.1,

\section{Acknowledgement}

I would like to thank two anonymous referees for carefully reading and giving many suggestions to improve the manuscript.

\section{References}

[1] N. D. Alikakos, $L^{p}$ bounds for solutions of reaction-diffusion equations, Comm. Partial Differential Equations 4, 827-868 (1979).

[2] N. Bellomo, A. Bellouquid, Y. Tao, M. Winkler, Toward a Mathematical Theory of Keller-Segel Models of Pattern Formation in Biological Tissues, Math. Models Methods Appl. Sci. 25, (2015).

[3] M. Chaplain, G. Lolas, Mathematical modelling of cancer invasion of tissue: dynamic hetergeneity. Net. Hetero. Med 1, 399-439 (2006).

[4] M. Hieber, J. Prüss, Heat kernels and maximal $l^{p}-l^{q}$ estimate for parabolic evolution equations, Comm. Partial Differential Equations 22, 1647-1669 (1997).

[5] T. Hillen, K.J. Painter, A user's guide to PDE models in a chemotaxis, J. Math. Biology 58, $183-217$ (2009). 
[6] D. Horstmann, From 1970 until present: The Keller-Segel model in chemotaxis and its consequences I, Jber. DMV 105 (3), 103-165 (2003).

[7] D. Horstmann, M. Winkler, Boundedness vs. blow-up in a chemotaxis system. J. Differential Equations 215, 52-107 (2005).

[8] K. Osaki, T. Tsujikawa, A. Yagi, M. Mimura, Exponential attractor for a chemotaxis-growth system of equations. Nonlinear Anal. 51, 119-144 (2002).

[9] E.F. Keller and L.A. Segel, Initiation of slime mold aggregation viewed as an instability. J. Theor. Biol., 26 399-415 (1970).

[10] T. Nagai, T. Senba, K. Yoshida, Application of the Trudinger-Moser inequality to a parabolic system of chemotaxis, Funkcial. Ekvac. 3, 411-433, (1997).

[11] C. Stinner, C. Surulescu, M. Winkler, Global weak solutions in a PDE-ODE system modeling multiscale cancer cell invasion, SIAM J. Math. Anal. 46, 1969-2007, (2014).

[12] Y. Tao, Global existence for a haptotaxis model of cancer invasion with tissue remodeling. Nonlinear Anal. 12(1), 418-435 (2011).

[13] Y. Tao, Boundedness in a two-dimensional chemotaxis-haptotaxis system. arXiv:1407.7382 (2014).

[14] Y. Tao, M, Wang, Global solution for a chemotactic-haptotactic model of cancer invasion. Nonlinearity 21, 2221-2238 (2008).

[15] Y. Tao, M. Winkler, Dominance of chemotaxis in a chemotaxis-haptotaxis model. Nonlinearity 27, 1225-1239 (2014).

[16] Y. Tao, M. Winkler, Energy-type estimates and global solvability in a two-dimensional chemotaxis-haptotaxis model with remodeling of non-diffusible attractant, J. Differential Equations 257, 784-815 (2014).

[17] Y. Tao, M. Winkler, Boundedness and stabilization in a multi-dimensional chemotaxis-haptotaxis model, Pro. Roy. Soc. Edingburgh, Sect. A 144, 1067-1087 (2014).

[18] J.I. Tello, M. Winkler, A chemotaxis system with logistic source. Commun. Partial Differential Equations 32 , 849-877 (2007).

[19] M. Winkler, Aggregation vs. global diffusive behavior in the higher-dimensional Keller-Segel model. J. Differential Equations, 248(12):2889-2905, (2010).

[20] M. Winkler, Boundedness in the higher-dimensional parabolic-parabolic chemotaxis system with logistic source. Comm. Partial Differential Equations 35, 1516-1537 (2010).

[21] M. Winkler, Finite-time blow-up in the higher-dimensional parabolic-parabolic Keller-Segel system, J. Math. Pures Appl. 100, 748-767 (2013). 